PROCEEDINGS OF THE

AMERICAN MATHEMATICAL SOCIETY

Volume 139, Number 4, April 2011, Pages 1411-1420

S 0002-9939(2010)10564-6

Article electronically published on September 1, 2010

\title{
CARATHÉODORY MEASURE HYPERBOLICITY AND POSITIVITY OF CANONICAL BUNDLES
}

\author{
SHIN KIKUTA \\ (Communicated by Franc Forstneric)
}

\begin{abstract}
In this paper, we prove that the curvature of the Carathéodory pseudo-volume form is bounded above by -1 . On the set where the pseudovolume form is non-degenerate, the curvature current of the singular Hermitian metric associated with the Carathéodory pseudo-volume form is proved to be strictly positive. Due to these curvature properties, we obtain an explicit relation between the Carathéodory measure hyperbolicity and the positivity of the canonical bundle. Moreover, we show a relation between the Carathéodory measure hyperbolicity, the existence of the Bergman kernel form and the existence of the Bergman metric.
\end{abstract}

\section{INTRODUCTION}

The positivity of the canonical bundle of a complex manifold is an important notion in algebraic geometry. In differential geometry, the positivity of the curvature of the pseudo-volume form associated to a complex manifold corresponds to the algebraic geometric positivity of the canonical bundle. The Bergman kernel form is used to prove the positivity of the canonical bundle in transcendental methods.

In this paper, we are concerned with the Kobayashi's curvature and the curvature current of the Carathéodory pseudo-volume form on a complex manifold. We clarify the relationship between the positivity of the Carathéodory pseudo-volume form and the positivity of the Bergman kernel form.

The Carathéodory pseudo-volume form is an important tool for studying holomorphic mappings between equidimensional complex manifolds. The definition of the Carathéodory pseudo-volume form $v_{X}^{C}$ on a complex manifold $X$ is analogous to that of the Carathéodory pseudo-metric. Regarding the pseudo-metric, Wong 10, Burbea 2 and Suzuki 9 showed that the holomorphic sectional curvature of the Carathéodory pseudo-metric is bounded above by -1 . Regarding the pseudo-volume form, henceforth denoted by $v$, Kobayashi [6] defined its curvature $K_{v}$ by

$$
K_{v}:=-\frac{2^{n}}{(n+1)^{n} n !} \frac{(\sqrt{-1} \partial \bar{\partial} \log v)^{n}}{v}
$$

on the open set where $v$ is $C^{2}$ and non-degenerate.

Received by the editors November 4, 2009 and, in revised form, April 20, 2010 and April 28, 2010 .

2010 Mathematics Subject Classification. Primary 32Q45; Secondary 32J18, 32J25.

Key words and phrases. Carathéodory pseudo-volume form, curvature, canonical bundle, Bergman kernel form.

(C)2010 American Mathematical Society 1411

Reverts to public domain 28 years from publication 
In Section 2, we define the curvature $K_{v_{X}^{C}}$ of the continuous pseudo-volume form $v_{X}^{C}$ analogously, by using the positivity of its curvature current $\Theta_{\left(v_{X}^{C}\right)^{-1}}:=$ $\sqrt{-1} \partial \bar{\partial} \log v_{X}^{C}$. Here, we regard $\left(v_{X}^{C}\right)^{-1}$ as a singular Hermitian metric on the canonical bundle $K_{X}$ of $X$. When $v_{X}^{C}$ is non-trivial, i.e., when $X$ is Carathéodory measure hyperbolic, we prove that, on a Carathéodory measure hyperbolic complex manifold, the curvature $K_{v_{X}^{C}}$ is bounded above by -1 and the curvature current $\sqrt{-1} \partial \bar{\partial} \log v_{X}^{C}$ is strictly positive on the open set where $v_{X}^{C}$ is non-degenerate.

Theorem 1.1. Let $X$ be a Carathéodory measure hyperbolic complex manifold. Then the curvature $K_{v_{X}^{C}}$ of the Carathéodory pseudo-volume form $v_{X}^{C}$ is bounded above by -1 , and the curvature current $\Theta_{\left(v_{X}^{C}\right)^{-1}}$ is strictly positive on the open set where $v_{X}^{C}$ is non-degenerate.

$\mathrm{Wu}$ [1] stated the second part of Theorem 1.1 without proof. We prove Theorem 1.1 in Section 3.

Thanks to Theorem 1.1 we show that Carathéodory measure hyperbolicity implies the positivity of the canonical bundle.

Corollary 1.2. Let $X$ be a compact complex manifold and $\tilde{X}$ its universal covering space.

Then we have

$$
\operatorname{vol}\left(K_{X}\right) \geq \frac{n !(n+1)^{n}}{(4 \pi)^{n}} \operatorname{vol}_{v_{\tilde{X}}^{C}}(X),
$$

where $\operatorname{vol}_{v_{\tilde{X}}^{C}}$ is the measure on $X$ with respect to $v_{\tilde{X}}^{C}$. Especially, if $\tilde{X}$ is Carathéodory measure hyperbolic, then $X$ is of general type.

If $\tilde{X}$ is strongly Carathéodory measure hyperbolic, i.e., $v_{\tilde{X}}^{C}$ is positive everywhere on $\tilde{X}$, then $X$ is projective algebraic with the ample canonical bundle.

To prove this result, we use another expression formula (Popovici [8]) of the volume of the canonical bundle

$$
\operatorname{vol}\left(K_{X}\right):=\limsup _{m \rightarrow \infty} n ! \frac{h^{0}\left(m K_{X}\right)}{m^{n}}
$$

with respect to the curvature currents and Richberg's regularization theorem. Corollary 1.2 shows that the Carathéodory measure hyperbolicity, namely, the positivity of the Carathéodory pseudo-volume form, leads explicitly to the positivity of the canonical bundle.

Remark that the Carathéodory pseudo-volume form and the Bergman kernel form are intrinsic: they depend only on the complex structure of $X$. Thanks to Corollary [1.2. we show that the Carathéodory measure hyperbolicity, i.e., the positivity of the Carathéodory pseudo-volume form, implies the positivity of the Bergman kernel form and of the Bergman metric.

Theorem 1.3. Assume that $\tilde{X}$ is a Carathéodory measure hyperbolic manifold with a smooth compact quotient. Then the Bergman kernel form is positive and the Bergman metric is strictly positive on the open set where the Carathéodory pseudo-volume form $v_{\tilde{X}}^{C}$ is positive.

In Section 4 we prove Theorem 1.3 by using the Carathéodory measure hyperbolicity and some $L^{2}$ estimates for the $\bar{\partial}$ operator (cf. Chen [3]). 


\section{Some BAsic properties AND Definition of the CURVATURE FOR THE CARATHÉODORY PSEUdO-VOLUME FORMS}

Henceforth, $X$ denotes a connected paracompact complex manifold.

Definition 2.1 (10]). The Carathéodory pseudo-volume form $v_{X}^{C}$ on a complex manifold $X$ is defined by

$$
v_{X}^{C}:=\sup \left\{f^{*} v_{1} ; f \in \operatorname{Hol}\left(X, \mathbb{B}^{n}\right)\right\},
$$

where $v_{1}:=2^{n}\left(1-|z|^{2}\right)^{-(n+1)} \bigwedge_{\alpha=1}^{n} d z^{\alpha} \wedge d \bar{z}^{\alpha}$ is the Poincaré volume form on the $n$-dimensional complex ball $\mathbb{B}^{n}$ and $\operatorname{Hol}\left(X, \mathbb{B}^{n}\right)$ is the space of all holomorphic mappings from $X$ to $\mathbb{B}^{n}$.

Since $\mathbb{B}^{n}$ is homogeneous, the Ascoli-Arzelà theorem tells us that, for any $x \in X$, there exists $f_{x} \in \operatorname{Hol}\left(X, \mathbb{B}^{n}\right)$ with $f_{x}(x)=o$ such that

$$
\left(v_{X}^{C}\right)_{x}=\sup \left\{\left(f^{*} v_{1}\right)_{x}: f \in \operatorname{Hol}\left(X, \mathbb{B}^{n}\right), f(x)=o\right\}=\left(f_{x}^{*} v_{1}\right)_{x} .
$$

Therefore the pseudo-volume form is continuous on $X$. Thus, $\mathrm{Z}\left(v_{X}^{C}\right):=\{x \in$ $\left.X ;\left(v_{X}^{C}\right)_{x}=0\right\}$ is a closed analytic subset of $X$.

We recall the definition of the curvature of the regular pseudo-volume forms:

Definition $2.2([10])$. For any pseudo-volume form $v$, we define the curvature $K_{v}$ by

$$
K_{v}:=-\frac{2^{n}}{(n+1)^{n} n !} \frac{(\sqrt{-1} \partial \bar{\partial} \log v)^{n}}{v},
$$

where the pseudo-volume form is non-degenerate and $C^{2}$.

Now, we want to define a notion of curvature for the Carathéodory pseudovolume form analogously. In general, however, the Carathéodory pseudo-volume form is not regular enough, not even continuous; thus we cannot apply this definition to $v_{X}^{C}$ directly. Nevertheless, since the logarithm of the Carathéodory pseudovolume form is plurisubharmonic, $\sqrt{-1} \partial \bar{\partial} \log v_{X}^{C}$ is a positive $(1,1)$-current. In particular, it is a normal current, i.e., a $(1,1)$-form whose coefficients are Radon measures. Therefore, we can take the absolutely continuous part of $\sqrt{-1} \partial \bar{\partial} \log v_{X}^{C}$ with respect to the Lebesgue measure. Denote it by $\left(\sqrt{-1} \partial \bar{\partial} \log v_{X}^{C}\right)_{\text {ac }}$. Its coefficients are locally integrable functions. We define the curvature $K_{v_{X}^{C}}$ of the Carathéodory pseudo-volume form by replacing $\sqrt{-1} \partial \bar{\partial} \log v_{X}^{C}$ with $\left(\sqrt{-1} \partial \bar{\partial} \log v_{X}^{C}\right)$ ac in the above definition:

Definition 2.3. The curvature $K_{v_{X}^{C}}$ of the Carathéodory pseudo-volume form $v_{X}^{C}$ is defined by

$$
K_{v_{X}^{C}}:=-\frac{2^{n}}{(n+1)^{n} n !} \frac{\left(\sqrt{-1} \partial \bar{\partial} \log v_{X}^{C}\right)_{\mathrm{ac}}^{n}}{v_{X}^{C}} .
$$

\section{Proof of Theorem 1.1}

We prove the first part of Theorem 1.1. It is sufficient to prove locally that

$$
\left(\sqrt{-1} \partial \bar{\partial} \log v_{X}^{C}\right)_{\mathrm{ac}}^{n} \geq \frac{(n+1)^{n} n !}{2^{n}} v_{X}^{C}
$$

almost everywhere on $X$. Hence we may consider that $v_{X}^{C}$ and $f_{x}^{*} v_{1}$ are non-negative functions. 
We fix a countable dense subset $\left\{x_{i}\right\}_{i \in \mathbb{N}} \subset X$ such that $v_{k}^{C}:=\max _{j=1,2, \ldots, k} f_{x_{j}}^{*} v_{1}$ $\stackrel{k \rightarrow \infty}{\longrightarrow} v_{X}^{C}$ on $X$, where the convergence is locally uniform. Now for any $k \in \mathbb{N}$, we take sequences of positive numbers $\left(\epsilon_{j}(l)\right)_{l \in \mathbb{N}}(j=1,2, \ldots, k)$ such that each $\left(\epsilon_{j}(l)\right)_{l \in \mathbb{N}}$ decreases monotonously to 0 . Furthermore, we require these sequences to satisfy the following property: for any $l \in \mathbb{N}$, the set defined by the equation $\log f_{x_{i}}^{*} v_{1}+\epsilon_{i}(l)=\log f_{x_{j}}^{*} v_{1}+\epsilon_{j}(l)$ is of Lebesgue measure zero for each $i, j \in$ $\{1,2, \ldots k\}$ with $i<j$. Finally, we also have the locally uniform convergence $\max _{j=1,2, \ldots, k}\left(\log f_{x_{j}}^{*} v_{1}+\epsilon_{j}(l)\right) \stackrel{l \rightarrow \infty}{\longrightarrow} \log v_{k}^{C}$ on $X$.

For each $i \in\{1,2, \ldots, k\}$, on the open set defined by the inequality $\log f_{x_{i}}^{*} v_{1}+$ $\epsilon_{i}(l)>\max _{j=1,2, \ldots, \hat{i}, \ldots, k}\left(\log f_{x_{j}}^{*} v_{1}+\epsilon_{j}(l)\right)$, we obtain, by setting $K_{v_{1}} \equiv-1$,

$$
\begin{aligned}
& \left(\sqrt{-1} \partial \bar{\partial} \max _{j=1,2, \ldots, k}\left(\log f_{x_{j}}^{*} v_{1}+\epsilon_{j}(l)\right)\right)^{n}=\left(\sqrt{-1} \partial \bar{\partial}\left(\log f_{x_{i}}^{*} v_{1}+\epsilon_{i}(l)\right)\right)^{n} \\
& =\left(\sqrt{-1} \partial \bar{\partial} \log f_{x_{i}}^{*} v_{1}\right)^{n} \geq f_{x_{i}}^{*}\left(\sqrt{-1} \partial \bar{\partial} v_{1}\right)^{n}=f_{x_{i}}^{*}\left(\frac{n !(n+1)^{n}}{2^{n}} v_{1}\right) \\
& \geq \frac{n !(n+1)^{n}}{2^{n}} \exp \left(\max _{j=1,2, \ldots, k}\left(\log f_{x_{j}}^{*} v_{1}+\epsilon_{j}(l)\right)-\max _{j=1,2, \ldots, k} \epsilon_{j}(l)\right) .
\end{aligned}
$$

Therefore, because of choice of the sequences $\left\{\left(\epsilon_{j}(l)\right)_{l \in \mathbb{N}}\right\}_{j=1,2, \ldots, k}$, we infer that

$$
\begin{aligned}
(\sqrt{-1} \partial \bar{\partial} & \left.\max _{j=1,2, \ldots, k}\left(\log f_{x_{j}}^{*} v_{1}+\epsilon_{j}(l)\right)\right)_{\mathrm{ac}}^{n} \\
& \geq \frac{n !(n+1)^{n}}{2^{n}} \exp \left(\max _{j=1,2, \ldots, k}\left(\log f_{x_{j}}^{*} v_{1}+\epsilon_{j}(l)\right)-\max _{j=1,2, \ldots, k} \epsilon_{j}(l)\right)
\end{aligned}
$$

almost everywhere on $X$.

To conclude, we need the following key lemma by Boucksom:

Lemma 3.1 ([1]). Let $\left(T_{k}\right)_{k \in \mathbb{N}}, T$ be positive $(1,1)$-currents on $X$. If $T_{k} \stackrel{k \rightarrow \infty}{\longrightarrow} T$, we have $\left(T_{\mathrm{ac}}\right)^{n} \geq \liminf _{k \rightarrow \infty}\left(T_{k}\right)_{\mathrm{ac}}^{n}$.

We apply this lemma with $T_{l}=\sqrt{-1} \partial \bar{\partial} \max _{j=1,2, \ldots, k}\left(\log f_{x_{j}}^{*} v_{1}+\epsilon_{j}(l)\right)$ and $T=$ $\sqrt{-1} \partial \bar{\partial} \max _{j=1,2, \ldots, k} \log f_{x_{j}}^{*} v_{1}$ to obtain

$$
\left(\sqrt{-1} \partial \bar{\partial} \log v_{k}^{C}\right)_{\mathrm{ac}}^{n} \geq \frac{n !(n+1)^{n}}{2^{n}} v_{k}^{C} .
$$

Applying this lemma once again with $T_{k}=\sqrt{-1} \partial \bar{\partial} \log v_{k}^{C}$ and $T=\sqrt{-1} \partial \bar{\partial} \log v_{X}^{C}$, we obtain the desired inequality:

$$
\left(\sqrt{-1} \partial \bar{\partial} \log v_{X}^{C}\right)_{\mathrm{ac}}^{n} \geq \frac{n !(n+1)^{n}}{2^{n}} v_{X}^{C} .
$$

We prove the second part of Theorem 1.1. It is sufficient to prove that for any $x_{0} \in X \backslash \mathrm{Z}\left(v_{X}^{C}\right)$, there exists a small compact neighborhood $K$ of $x_{0}$ in a holomorphic chart $\left(U ; z^{1}, z^{2}, \ldots, z^{n}\right)$ centered at $x_{0}$ such that $\sqrt{-1} \partial \bar{\partial} \log v_{X}^{C}$ is strictly positive there.

For a compact neighborhood $K$ of $x_{0}$ in $\left(U ; z^{1}, z^{2}, \ldots, z^{n}\right)$, set

$$
\mathcal{K}:=\left\{f_{x} \in \operatorname{Hol}\left(X, \mathbb{B}^{n}\right) ; x \in K\right\} .
$$


Then, we have $\log v_{X}^{C} \mid K=\sup _{f \in \mathcal{K}} \log f^{*} v_{1}$, and for any $f \in \mathcal{K}$, the inequality

$$
\begin{aligned}
& \sqrt{-1} \partial \bar{\partial} \log f^{*} v_{1} \\
\geq & \sqrt{-1}(n+1)\left\{\frac{\left(1-|f(z)|^{2}\right) \delta_{i \bar{j}}+\overline{f^{i}}(z) f^{j}(z)}{\left(1-|f(z)|^{2}\right)^{2}}\right\} \frac{\partial f^{i}}{\partial z^{\alpha}} \frac{\overline{\partial f^{j}}}{\partial z^{\beta}} d z^{\alpha} \wedge d \overline{z^{\beta}}
\end{aligned}
$$

holds true.

Next we prove two key lemmas regarding the compactness of $\mathcal{K}$ and the existence of an appropriate neighbourhood $K$.

Lemma 3.2. $\mathcal{K}$ is compact in $C\left(X, \mathbb{B}^{n}\right)$.

Proof. By the complete Kobayashi hyperbolicity of $\mathbb{B}^{n}$ and the compactness of $K$, $\mathcal{K}$ is relatively compact in $C\left(X, \mathbb{B}^{n}\right)$.

Let $\left(f_{x_{i}}\right)_{i \in \mathbb{N}} \subset \mathcal{K}$, i.e.,$\left\{x_{i}\right\}_{i \in \mathbb{N}} \subset K$ with $f_{x_{i}} \stackrel{i \rightarrow \infty}{\longrightarrow} f_{\infty}$ in $C\left(X, \mathbb{B}^{n}\right)$ for some $f_{\infty} \in \operatorname{Hol}\left(X, \mathbb{B}^{n}\right)$. By the compactness of $K$, there exists a subsequence $\left(x_{i(k)}\right)_{k \in \mathbb{N}}$ of $\left(x_{i}\right)_{i \in \mathbb{N}}$ such that $x_{i(k)} \stackrel{k \rightarrow \infty}{\longrightarrow} x_{\infty}$ for some $x_{\infty} \in K$. Then $f_{\infty}\left(x_{\infty}\right)=o$ and, since $v_{X}^{C}$ is continuous, we obtain

$$
\left(v_{X}^{C}\right)_{x_{\infty}}=\lim _{k \rightarrow \infty}\left(v_{X}^{C}\right)_{x_{i(k)}}=\lim _{k \rightarrow \infty}\left(f_{x_{i(k)}}^{*} v_{1}\right)_{x_{i(k)}}=\left(f_{\infty}^{*} v_{1}\right)_{x_{\infty}}
$$

Hence $\mathcal{K}$ is closed in $C\left(X, \mathbb{B}^{n}\right)$ and is thus compact.

Lemma 3.3. There exists $K$, small enough, such that, for some constant $C>0$,

$$
\left|\left(\sum_{\beta=1}^{n} \frac{\partial f^{i}}{\partial z^{\beta}}(z) \zeta^{\beta}\right)_{i=1,2, \ldots, n}\right| \geq C|\zeta|
$$

holds for all $z \in K, f \in \mathcal{K}$ and $\zeta=\left(\zeta^{\beta}\right)_{\beta=1,2, \ldots, n} \in \mathbb{C}^{n}$.

Proof. We use reductio ad absurdum. Suppose that, for any $\epsilon>0$, there exist $z_{\epsilon}, w_{\epsilon} \in U$ and $\zeta_{\epsilon} \in \mathbb{C}^{n}$ with $\left|\zeta_{\epsilon}\right|=1$ such that $z_{\epsilon} \stackrel{\epsilon \rightarrow 0}{\longrightarrow} x_{0}, w_{\epsilon} \stackrel{\epsilon \rightarrow 0}{\longrightarrow} x_{0}$ and

$$
\left|\left(\sum_{\beta=1}^{n} \frac{\partial f_{z_{\epsilon}}^{i}}{\partial z^{\beta}}\left(w_{\epsilon}\right)\left(\zeta_{\epsilon}\right)^{\beta}\right)_{i=1,2, \ldots, n}\right| \leq \epsilon .
$$

Then, by using the convergent subsequences of $z_{\epsilon}, w_{\epsilon}, f_{z_{\epsilon}}$ and $\zeta_{\epsilon}$ as in the proof of Lemma 3.2. we can find $f_{x_{0}}$ and $\zeta_{0} \in \mathbb{C}^{n}$ with $\left|\zeta_{0}\right|=1$ such that

$$
\left|\left(\sum_{\beta=1}^{n} \frac{\partial f_{x_{0}}^{i}}{\partial z^{\beta}}\left(x_{0}\right)\left(\zeta_{0}\right)^{\beta}\right)_{i=1,2, \ldots, n}\right|=0 .
$$

This contradicts $\left(v_{X}^{C}\right)_{x_{0}}=\left(f_{x_{0}}^{*} v_{1}\right)_{x_{0}} \neq 0$.

We now fix a compact set $K$ as in Lemma 3.3. Since $\left\{f(z) \in \mathbb{B}^{n} ; f \in \mathcal{K}, z \in K\right\}$ is compact by Lemma 3.2. we infer from (3.1) that there exists some constant $C^{\prime}>0$ 
such that

$$
\begin{aligned}
(n+1)\left\{\frac{\left(1-|f(z)|^{2}\right) \delta_{i \bar{j}}+\overline{f^{i}}(z) f^{j}(z)}{\left(1-|f(z)|^{2}\right)^{2}}\right\} \frac{\partial f^{i}}{\partial z^{\alpha}} \frac{\overline{\partial f^{j}}}{\partial z^{\beta}} \zeta^{\alpha} \overline{\zeta^{\beta}} \\
\geq C^{\prime}\left|\left(\sum_{\beta=1}^{n} \frac{\partial f^{i}}{\partial z^{\beta}}(z) \zeta^{\beta}\right)_{i=1,2, \ldots, n}\right|^{2}
\end{aligned}
$$

for any $z \in K, f \in \mathcal{K}$ and $\zeta=\left(\zeta^{\beta}\right)_{\beta=1,2, \ldots, n} \in \mathbb{C}^{n}$. Moreover, it follows from (3.2) and Lemma 3.3 that

$$
(n+1)\left\{\frac{\left(1-|f(z)|^{2}\right) \delta_{i \bar{j}}+\overline{f^{i}}(z) f^{j}(z)}{\left(1-|f(z)|^{2}\right)^{2}}\right\} \frac{\partial f^{i}}{\partial z^{\alpha}}(z) \frac{\overline{\partial f^{j}}}{\partial z^{\beta}}(z) \zeta^{\alpha} \overline{\zeta^{\beta}} \geq c|\zeta|^{2}
$$

for all $z \in K, f \in \mathcal{K}$ and $\zeta=\left(\zeta^{\beta}\right)_{\beta=1,2, \ldots, n} \in \mathbb{C}^{n}$, where we have set $c:=C^{2} \cdot C^{\prime}$. These inequalities altogether imply

$$
\sqrt{-1} \partial \bar{\partial} \log v_{X}^{C} \geq c \sqrt{-1} \partial \bar{\partial}|z|^{2}
$$

on $K$.

Finally, we prove Corollary [1.2. Regarding the first part of this corollary, we combine the first part of Theorem 1.1 with a formula for the volume of a line bundle $L$ by Boucksom and Popovici:

Theorem 3.4 ([1, [8]). Let $X$ be a compact complex manifold and $L$ a holomorphic line bundle over $X$. Then we have

$$
\operatorname{vol}(L)=\sup \left\{\int_{X} T_{\mathrm{ac}}^{n} ; T \in c_{1}(L) \text { is a positive }(1,1) \text {-current }\right\} .
$$

We will apply this result with $L=K_{X}$. First, note that the Carathéodory pseudo-volume form $v_{\tilde{X}}^{C}$ of the universal covering $\tilde{X}$ on $X$ is biholomorphically invariant. Thus, we can regard $\left(v_{\tilde{X}}^{C}\right)^{-1}$ as a singular Hermitian metric on $K_{X}$. Hence we can take $T=(2 \pi)^{-1} \Theta_{\left(v_{\tilde{X}}^{C}\right)^{-1}} \in c_{1}\left(K_{X}\right)$ in Theorem 3.4. From the first part of Theorem 1.2, we conclude that

$$
\operatorname{vol}\left(K_{X}\right) \geq \int_{X}\left(\frac{1}{2 \pi} \sqrt{-1} \partial \bar{\partial} \log v_{X}^{C}\right)_{\mathrm{ac}}^{n} \geq \frac{n !(n+1)^{n}}{(4 \pi)^{n}} \operatorname{vol}_{v_{\bar{X}}^{C}}(X) .
$$

To prove the second part of this corollary, we apply Richberg's regularization technique ([5]) to the continuous strictly plurisubharmonic function $\log v_{\tilde{X}}^{C}$. We use this to regularize the singular Hermitian metric $\left(v_{\tilde{X}}^{C}\right)^{-1}$ while keeping the strict positivity of the curvature current. Hence we get a smooth Hermitian metric on $K_{X}$ that has the strictly positive curvature form on $X$. By Kodaira's embedding theorem, $X$ turns out to be a projective algebraic manifold with ample $K_{X}$.

\section{Proof of Theorem 1.3}

We denote by $(\alpha, \beta):=\sqrt{-1}^{n^{2}} \int_{X} \alpha \wedge \bar{\beta}$ the Hermitian inner product of two $(n, 0)$-forms $\alpha, \beta$. Recall the definitions of the Bergman kernel and metric:

Definition 4.1. Let $X$ be a paracompact connected complex manifold and $\left\{\alpha_{i}\right\}_{i=0}^{\infty}$ be a complete orthonormal system of the Hilbert space $L^{2}\left(\Omega_{X}^{n}\right)$ consisting of the 
holomorphic $n$-form $\alpha$ satisfying $\|\alpha\|^{2}=(\alpha, \alpha)<\infty$. The Bergman kernel $v_{X}^{B}$ on $X$ is defined by

$$
v_{X}^{B}:=\sum_{i=0}^{\infty} \sqrt{-1} n^{2} \alpha_{i} \wedge \overline{\alpha_{i}}=\sup _{\alpha \in L^{2}\left(\Omega_{X}^{n}\right),\|\alpha\|=1} \sqrt{-1} n^{2} \alpha \wedge \bar{\alpha} .
$$

The pseudo-metric associated to the curvature form $\Theta_{\left(v_{X}^{B}\right)^{-1}}$ of the singular Hermitian metric $\left(v_{X}^{B}\right)^{-1}$ as the fundamental $(1,1)$-form is called the Bergman pseudometric on $X$. When $\Theta_{\left(v_{X}^{B}\right)^{-1}}$ is non-degenerate on $X$, we call it the Bergman metric on $X$.

To prove Theorem 1.3. we start by using some $L^{2}$ estimates for the $\bar{\partial}$ operator in order to construct the suitable elements in $L^{2}\left(\Omega_{X}^{n}\right)$ by following Chen [3].

Let $\tilde{X}$ be a Galois covering of a compact complex manifold $X$ and let $\pi_{X}$ : $\tilde{X} \rightarrow X$ be the covering map. Note that $v_{\tilde{X}}^{C}$ is biholomorphically invariant, and we can consider it as defined on $X$. We proceed to construct the weight function and complete Kähler domain in $\tilde{X}$ to set up the suitable $L^{2}$ space in order to use the $L^{2}$ estimate for the $\bar{\partial}$ operator.

First we construct the weight function. Let $\left\{x_{i}\right\}_{i \in \mathbb{N}} \subset \tilde{X}$ be a countable dense subset such that

$$
\tilde{X} \backslash Z\left(v_{\tilde{X}}^{C}\right)=\bigcup_{i=1}^{\infty} U_{i}, \quad U_{i}:=\tilde{X} \backslash\left(d f_{x_{i}}^{1} \wedge d f_{x_{i}}^{2} \wedge \cdots \wedge d f_{x_{i}}^{n}\right)^{-1}(0)
$$

for $f_{x_{i}}=\left(f_{x_{i}}^{1}, \ldots, f_{x_{i}}^{n}\right) \in \operatorname{Hol}\left(\tilde{X}, \mathbb{B}^{n}\right)$ in the notation of Section 2. Consider the smooth and bounded plurisubharmonic function

$$
\phi:=\log \left(1+\sum_{i=1}^{\infty} \frac{1}{2^{i}}\left\|f_{x_{i}}\right\|^{2}\right),
$$

where $\left\|f_{x_{i}}\right\|^{2}:=\sum_{j=1}^{n}\left|f_{x_{i}}^{j}\right|^{2}<1$.

Lemma 4.2. $\phi$ is strictly plurisubharmonic on $\tilde{X} \backslash Z\left(v_{\tilde{X}}^{C}\right)$.

Proof. We denote by $g_{k}(k=0,1,2, \ldots)$ the bounded holomorphic functions in the definition of $\phi$ as they come ordered in the sum: $g_{0}=1, g_{1}=\frac{1}{2} f_{x_{1}}^{1}, g_{2}=$ $\frac{1}{2} f_{x_{1}}^{2}, \ldots, g_{n+1}=\frac{1}{2} f_{x_{1}}^{n}, g_{n+2}=\frac{1}{2^{2}} f_{x_{2}}^{1}$, and so on. We set $\log \kappa:=\log \left(\sum_{k=0}^{\infty}\left|g_{k}\right|^{2}\right)$. Now, take any point $x \in \tilde{X} \backslash Z\left(v_{\tilde{X}}^{C}\right)$ and let $i \in \mathbb{N}$ such that $x \in U_{i}$. By the definition of $f_{x}$ and $U_{i},\left(z^{\alpha}\right)_{\alpha=1,2, \ldots, n}=\left(f_{x_{i}}^{\alpha}\right)_{\alpha=1,2, \ldots, n}$ is a holomorphic local coordinate system around $x$ of $\tilde{X}$. The complex Hessian with respect to this coordinate system is

$$
\frac{\partial^{2} \phi}{\partial z^{\alpha} \partial \overline{z^{\beta}}}=\frac{1}{\kappa^{2}} \sum_{k<l}^{\infty}\left\{g_{k} \frac{\partial g_{l}}{\partial z^{\alpha}}-\frac{\partial g_{k}}{\partial z^{\alpha}} g_{l}\right\} \overline{\left\{g_{k} \frac{\partial g_{l}}{\partial z^{\beta}}-\frac{\partial g_{k}}{\partial z^{\beta}} g_{l}\right\}} .
$$

Therefore for any $\zeta=\left(\zeta^{\alpha}\right)_{\alpha=1,2, \ldots, n} \in \mathbb{C}^{n}$ we obtain

$$
\sum_{\alpha, \beta=1}^{n} \frac{\partial^{2} \phi}{\partial z^{\alpha} \partial \overline{z^{\beta}}}(x) \zeta^{\alpha} \overline{\zeta^{\beta}}=\sum_{k<l}^{\infty}\left|\sum_{\alpha=1}^{n}\left\{g_{k}(x) \frac{\partial g_{l}}{\partial z^{\alpha}}(x)-\frac{\partial g_{k}}{\partial z^{\alpha}}(x) g_{l}(x)\right\} \frac{\zeta^{\alpha}}{\kappa(x)}\right|^{2},
$$

which is equal to 0 if and only if

$$
\sum_{\alpha=1}^{n}\left\{g_{k}(x) \frac{\partial g_{l}}{\partial z^{\alpha}}(x)-\frac{\partial g_{k}}{\partial z^{\alpha}}(x) g_{l}(x)\right\} \zeta^{\alpha}=0
$$


for any $k, l=0,1, \ldots$ If, in the last expression, for any $\beta=1,2, \ldots, n$ we take $l=0$ and $k \in \mathbb{N}$ such that $g_{k}=\frac{1}{2^{i}} f_{x_{i}}^{\beta}, f_{x_{i}}^{\beta}=z^{\beta}$ holds, then $\zeta^{\beta}$ vanishes because of the choice of the local coordinate system $\left(z^{\alpha}\right)_{\alpha=1,2, \ldots, n}=\left(f_{x_{i}}^{\alpha}\right)_{\alpha=1,2, \ldots, n}$. Therefore the Hermitian form (4.1) vanishes if and only if $\zeta=0$, which means that $\phi$ is strictly plurisubharmonic at any $x \in \tilde{X} \backslash Z\left(v_{\tilde{X}}^{C}\right)$.

Second, we construct a complete Kähler domain in $\tilde{X}$. By hypothesis, $\tilde{X}$ has a smooth compact quotient complex manifold $X$, and by Corollary 1.2, $X$ is of general type. Hence Moishezon's theorem ([7]) implies that there exists a projective manifold $Y$ obtained after finitely many blow-ups $\sigma: Y=Y_{N} \stackrel{\sigma_{N}}{\rightarrow} Y_{N-1} \cdots Y_{2} \stackrel{\sigma_{2}}{\rightarrow}$ $Y_{1} \stackrel{\sigma_{1}}{\rightarrow} Y_{0}=X$ along smooth centers $S_{k} \subset Y_{k}$ for all $k=0,1, \ldots, N-1$. Denote by $E_{k+1}$ the divisor on $Y_{k+1}$ and by $S_{k}$ the subvariety of $Y_{k}$ with $\operatorname{codim}_{Y_{k}} S_{k} \geq 2$ such that $\sigma_{k+1}: Y_{k+1} \backslash E_{k+1} \stackrel{\sim}{\rightarrow} Y_{k} \backslash S_{k}$ is biholomorphic for any $k=0,1, \ldots, N-1$. Set $\left(\pi_{X}\right)^{-1}\left(S_{0}\right)$ by $\tilde{S}_{0}$. We see that the blow-up $\tilde{Y}_{1} \stackrel{\tilde{\sigma}_{4}}{\rightarrow} \tilde{X}$ along $\tilde{S}_{0}$ is the covering space $\pi_{Y_{1}}: \tilde{Y}_{1} \rightarrow Y_{1}$ over $Y_{1}$ satisfying the commutative diagram

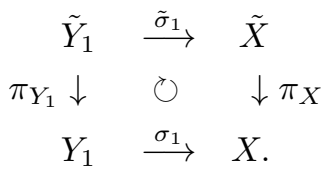

By iterating this process, we obtain the following commutative diagram:

$$
\begin{array}{rllllllll}
\tilde{\sigma}: \tilde{Y}=\tilde{Y}_{N} & \stackrel{\tilde{\sigma}_{N}}{\longrightarrow} & \tilde{Y}_{N-1} & \rightarrow & \cdots & \rightarrow & \tilde{Y}_{1} & \stackrel{\tilde{\sigma}_{1}}{\longrightarrow} & \tilde{X} \\
\pi_{Y} \downarrow & \circlearrowright & \downarrow \pi_{Y_{N-1}} & \cdots & \pi_{Y_{1}} \downarrow & \circlearrowright & \downarrow \pi_{X} \\
\sigma: Y & \stackrel{\sigma_{N}}{\longrightarrow} & Y_{N-1} & \rightarrow & \cdots & \rightarrow & Y_{1} & \stackrel{\sigma_{1}}{\longrightarrow} & X .
\end{array}
$$

The maps in rows are the blow-ups; the maps in columns are the coverings. Now, we can take a divisor $E$ on $Y$ and an analytic subset $S$ of $X$ of $\operatorname{codim}_{X} S \geq 2$. Set $\tilde{E}:=\left(\pi_{Y}\right)^{-1}(E)$ and $\tilde{S}:=\left(\pi_{X}\right)^{-1}(S)$. We obtain the commutative diagram

$$
\begin{aligned}
& \tilde{Y} \backslash \tilde{E} \quad \stackrel{\tilde{\sigma}}{\longrightarrow} \tilde{X} \backslash \tilde{S} \\
& \pi_{Y} \downarrow \quad \circlearrowright \quad \downarrow \pi_{X} \\
& Y \backslash E \stackrel{\sigma}{\longrightarrow} X \backslash S .
\end{aligned}
$$

The horizontal maps are biholomorphic.

By the biholomorphic invariance of the Carathéodory pseudo-volume form and diagram (4.2), we have

$$
\left.\tilde{\sigma}^{*} v_{\tilde{X}}^{C}\right|_{\tilde{Y} \backslash \tilde{E}}=\tilde{\sigma}^{*} v_{\tilde{X} \backslash \tilde{S}}^{C}=v_{\tilde{Y} \backslash \tilde{E}}^{C}=\left.v_{\tilde{Y}}^{C}\right|_{\tilde{Y} \backslash \tilde{E}} .
$$

Here we have used the Riemann extension theorem in the first and last equalities. Since the Carathéodory pseudo-volume form is continuous, we have $\tilde{\sigma}^{*} v_{\tilde{X}}^{C}=v_{\tilde{Y}}^{C}$. Since $Z\left(v_{\tilde{Y}}^{C}\right)=\tilde{E} \cup \tilde{\sigma}^{-1}\left(Z\left(v_{\tilde{X}}^{C}\right)\right)$, the map

$$
\tilde{\sigma}: \tilde{Y} \backslash Z\left(v_{\tilde{Y}}^{C}\right) \stackrel{\sim}{\rightarrow} \tilde{X} \backslash\left(\tilde{S} \cup Z\left(v_{\tilde{X}}^{C}\right)\right)
$$

is a biholomorphism.

The domain $D:=\tilde{X} \backslash\left(\tilde{S} \cup Z\left(v_{\tilde{X}}^{C}\right)\right)$ is the desired complete Kähler domain in $\tilde{X}$. To prove this, it is sufficient by (4.3) to prove that $\tilde{Y} \backslash Z_{\tilde{Y}}^{C}$ has a complete Kähler metric. This follows by a similar construction of Demailly [4, p. 471, Lemma 7.2]. 
Lemma 4.3. $\tilde{Y} \backslash Z_{\tilde{Y}}^{C}$ has a complete Kähler metric $\omega_{Y}$.

Proof. We observe that $\tilde{Y}$ has a complete Kähler metric $\omega_{0}$. Furthemore, there exists a smooth Hermitian metric $h$ on $K_{\tilde{Y}}$ satisfying $\omega_{0} \geq \Theta_{h}$ and the invariance by the covering transformation. By using $h$, we construct an upper continuous function $\tau$ by

$$
\tau:=\log \left(\sum_{i=1}^{\infty} \frac{1}{2^{i}} \tilde{h}\left(d f_{x_{i}}^{1} \wedge \cdots \wedge d f_{x_{i}}^{n}, d f_{x_{i}}^{1} \wedge \cdots \wedge d f_{x_{i}}^{n}\right)\right),
$$

where $\tau$ is well defined since $\left\|f_{x}\right\|<1$. Notice that $\tau$ is bounded by above. We may assume, without loss of generality, that it is smaller than or equal to -1 . We remark that $\tau^{-1}(-\infty)=Z\left(v_{\tilde{Y}}^{C}\right)$ and $\sqrt{-1} \partial \bar{\partial} \tau \geq-\Theta_{h} \geq-\omega_{0}$. Therefore we can construct a complete Kähler metric $\omega$ on $\tilde{Y} \backslash Z\left(v_{\tilde{Y}}^{C}\right)$ using $\tau$ and $\omega_{0}$ by the same argument as Demailly in [4, p. 471, Lemma 7.2]: it suffices to set $\omega_{\tilde{Y}}:=$ $3 \omega_{0}+\sqrt{-1} \partial \bar{\partial}(\tau-\log (-\tau))$.

To complete the proof, we use the next result by Chen in [3] applied to the complete Kähler manifold $\left(D, \omega_{D}:=\left(\left(\tilde{\sigma} \mid \tilde{Y} \backslash Z_{\tilde{Y}}^{C}\right)^{-1}\right)^{*} \omega_{Y}\right)$ :

Proposition 4.4. Let $\left(M, \omega_{M}\right)$ be a complete Kähler manifold. Suppose that there exists on $M$ a bounded smooth strictly plurisubharmonic function $\phi$. Then $M$ possesses a Bergman kernel and metric.

This proposition directly implies the existence of the Bergman kernel and metric on $D=\tilde{X} \backslash\left(\tilde{S} \cup Z\left(v_{\tilde{X}}^{C}\right)\right)$. Regarding the existence on $\tilde{X} \backslash Z\left(v_{\tilde{X}}^{C}\right)$, we observe that the proof of Proposition 4.4 turns out to be applicable to every point in $\tilde{S} \backslash Z\left(v_{\tilde{X}}^{C}\right)$ since the $\phi$ constructed above is strictly plurisubharmonic there. The existence of the Bergman kernel and of the Bergman metric on $\tilde{X} \backslash Z\left(v_{\tilde{X}}^{C}\right)$ follows.

\section{ACKNOWLEDGEMENTS}

The author would like to express his thanks to Professors Shigetoshi Bando and Hajime Tsuji for their kindness and valuable advice.

\section{REFERENCES}

1. S. Boucksom, On the volume of a line bundle, Internat. J. Math. 13 (2002), no. 10, 1043-1063. MR.1945706 (2003j:32025)

2. J. Burbea, On the Hessian of the Carathéodory metric, Rocky Mountain J. Math. 8 (1978), no. 3, 555-559. MR0481122(58:1268)

3. B.-Y. Chen, The Bergman metric on complete Kähler manifolds, Math. Ann. 327 (2003), no. 2, 339-349. MR2015074 (2004h:32023)

4. J.-P. Demailly, Complex analytic and differential geometry, www.agbook.ps.gz.

5. _ Regularization of closed positive currents and intersection theory, J. Algebraic Geom. 1 (1992), no. 3, 361-409. MR1158622 (93e:32015)

6. S. Kobayashi, Hyperbolic complex spaces, Grundlehren der Mathematischen Wissenschaften [Fundamental Principles of Mathematical Sciences], vol. 318, Springer-Verlag, Berlin, 1998. MR.1635983 (99m:32026)

7. X. Ma and G. Marinescu, Holomorphic Morse inequalities and Bergman kernels, Progress in Mathematics, vol. 254, Birkhäuser Verlag, Basel, 2007. MR2339952 (2008g:32030)

8. D. Popovici, Regularization of currents with mass control and singular Morse inequalities, J. Differential Geom. 80 (2008), no. 2, 281-326. MR.2454895 (2010d:32016)

9. M. Suzuki, The intrinsic metrics on the domains in $\mathbf{C}^{n}$, Math. Rep. Toyama Univ. 6 (1983), 143-177. MR719152 (86a:32051) 
10. B. Wong, On the holomorphic curvature of some intrinsic metrics, Proc. Amer. Math. Soc. 65 (1977), no. 1, 57-61. MR 0454081 (56:12332)

11. $\mathrm{H}$. Wu, Old and new invariant metrics on complex manifolds, Several complex variables (Stockholm, 1987/1988), Math. Notes, vol. 38, Princeton Univ. Press, Princeton, NJ, 1993, 640-682. MR1207887 (94a:32038)

Mathematical Institute, Tohoku University, Sendai 980-8578, JaPan

E-mail address: sa6m15@math.tohoku.ac.jp 